\title{
Successful Treatment of Chronic Thromboembolic Pulmonary Hypertension with Inhaled Nitric Oxide after Right Ventricular Thrombectomy
}

We report a case of a 42-year-old male with chronic thromboembolic pulmonary hypertension. His preoperative examination revealed severe hypoxemia $\left(\mathrm{PaO}_{2} 48 \mathrm{mmHg}, \mathrm{PaCO}_{2} 34 \mathrm{mmHg}\right.$ in room air), a mass in the right ventricle and severe pulmonary hypertension (pulmonary arterial pressure 70/33 $\mathrm{mmHg}$ ). We successfully performed right ventricular thrombectomy to prevent further embolization from the right ventricular thrombus. Using inhaled low dose nitric oxide (NO) during perioperative period, weaning from cardiopulmonary bypass and ventilator were easily done. In this case, inhaled NO was successfully administered for the perioperative management of chronic pulmonary hypertension. (Jpn J Thorac Cardiovasc Surg 2004; 52: 257-260)

Key words: pulmonary thromboembolism, chronic thromboembolic hypertension, inhaled nitric oxide

Kiyoaki Takaba, MD, Masaki Aota, MD, Michihito Nonaka, MD, Akihiro Sugimoto, MD, and Yutaka Konishi, MD.

I n 1987, nitric oxide (NO) was reported to be an important factor accounting for the biologic activity of endothelium derived relaxing factor. ${ }^{1}$ Inhaled nitric oxide (iNO) is an agent known to reduce pulmonary vascular resistance without systemic hypotension. Although successful iNO treatment of patients with pulmonary hypertension has been reported, clinical use of iNO as an adjunct to surgical treatment is not widespread. $^{2}$ The present report documents our successful surgical treatment of pulmonary hypertension ( $\mathrm{PH}$ ) after right ventricular thrombectomy.

\section{Case}

A 42-year-old man was admitted to our hospital with dyspnea. About one month before he was admitted to our hospital, he had had a sudden onset of chest pain

From Department of Cardiovascular Surgery, Japanese Red Cross Society Wakayama Medical Center, Wakayama, Japan.

Received for publication July 1, 2003.

Accepted for publication December 17, 2003.

Address for reprints: Kiyoaki Takaba, MD, Department of Cardiovascular Surgery, Japanese Red Cross Society Wakayama Medical Center, 4-20 Komatsubaradouri, Wakayama 640-8558, Japan. and dyspnea. With dyspnea progressively worsening, he visited a local physician. Transthoracic echocardiography at his doctor's office revealed a mobile mass in the dilated right ventricle with severe tricuspid valve regurgitation, and he was referred to our hospital for surgical treatment of cardiac tumor to prevent further embolization in the right ventricle. On admission, the degree of dyspnea corresponded to NYHA class III. His blood pressure was $126 / 79 \mathrm{mmHg}$ and his heart rate was regular, 74 beats/min at the time of admission. Cardiac auscultation showed decreased $\mathrm{S}_{2}$, and no murmur was heard. He had peripheral cyanosis, but no clubbed fingers. The liver was palpable at two fingerbreadths below the costal margin. Neither his leg showed varicose veins. The results of arterial blood gas analysis were $\mathrm{pH} 7.378, \mathrm{PaO}_{2} 48.7 \mathrm{mmHg}$, $\mathrm{PaCO}_{2} 34.2 \mathrm{mmHg}$ and $\mathrm{BE}-5.5$ in room air. A chest $\mathrm{X}$-ray showed mild cardiomegaly with a cardiothoracic ratio of $55 \%$, and prominence of the right atrium. An electrocardiogram showed normal sinus rhythm and right ventricular hypertrophy by voltage criteria. Transthoracic and transesophageal echocardiography showed the following findings: a mass arising from interventricular the septum and apex in the right ventricle, enlarged right atrium and ventricle with global hypokinesis in the right ventricle, grade III tricuspid regurgitation (the estimated RV pressure by regurgitant 
jet was about $70 \mathrm{mmHg}$ in systole), and remarkable shift of the interventricular septum to the left ventricle (Fig. 1). Pulmonary perfusion scan revealed a few defects $\left(S_{1+2}\right.$ in the left upper lobe, $S_{3}$ in the right upper lobe). Computed tomography demonstrated insufficient data to confirm the diagnosis or determine the optimum surgical strategy. No deep venous thrombosis was detected by sonography of bilateral lower legs. Cardiac catheterization was performed, pressure study of right heart catherization showed the following: PAP 70/33 (45) mmHg, PCW 6 mmHg, RVP 69/6 (7) $\mathrm{mmHg}, \mathrm{CO} 4.04 \mathrm{~L} / \mathrm{min}, \mathrm{CI} 2.31 \mathrm{~L} / \mathrm{min} / \mathrm{m}^{2}$, PVR 772.8 dyne $/ \mathrm{sec} / \mathrm{cm}^{5}$. Right ventriculograpghy showed no intracavitary filling defect. In coronary angiography, neither a feeding artery to the mass nor vascular blush was found. Hematological testing showed mild liver dysfunction, but no other specific abnormalities such as aniphospholipid antibody positive. On these findings, we undertook surgery to prevent further embolization in the right ventricular tumor.

Through median sternotomy, we performed mild hypothermic total cardiopulmonary bypass (CPB) between the ascending aorta and both vena cavae. The heart was arrested with antegrade tepid blood cardioplegia after aortic cross-clamping. Through right atriotomy and the tricuspid valve, a massive organized (partially fresh) thrombus was found in the apex of the right ventricle beneath the eccentric enlarged trabeculae, so we removed this thrombus as completely as possible. A piece of the mass was taken for cytological analysis. It had no malignancy and was only thrombus. Then the pulmonary trunk was incised longitudinally and we tried to remove the thrombi with direct suction, both pleural cavities were opened and the peripheral vascular bed was cleared by gentle manual compression of lungs, but no thrombi was detected. The annulus of tricuspid valve was enlarged, so we performed tricuspid annular plasty using a Carpenter Edwards $30 \mathrm{~mm}$ ring. The right atriotomy was closed and lung biopsy was performed from the left lung lingula. Histological examination demonstrated findings compatible with chronic thromboembolic pulmonary hypertension (Fig. 2).

Hemodynamic monitoring was performed with a Swan-Gantz catheter. NO was delivered continuously into the inspiratory limb of the ventilator and the inhaled concentration of NO was measured with a chemiluminescence analyzer. We started to administer iNO at $10 \mathrm{ppm}$ at the time of anesthetic induction to prevent $\mathrm{PH}$ crisis. Intubation and anesthtetic induction was performed uneventfully. When cardiopulmonary bypass was instituted, we discontinued iNO adminis-
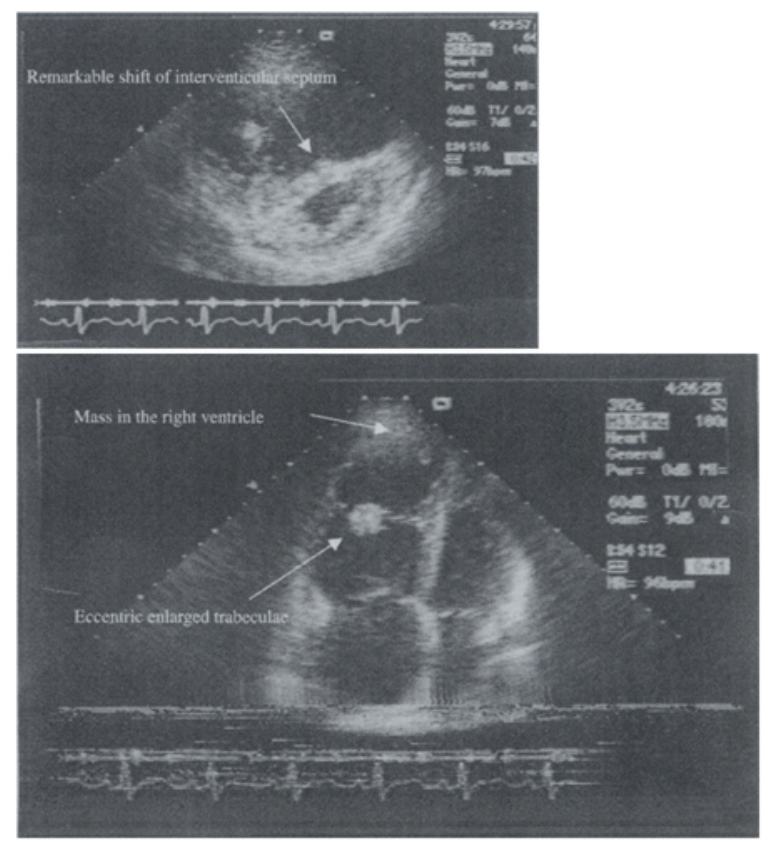

Fig. 1. Echocardiography showed findings as follows: a mass in the right ventricle, enlarged right atrium and ventricle, and remarkable shift of the interventricular septum to the left ventricle.

tration. Weaning from cardiopulmonary bypass was poorly tolerated, so we inserted IABP via the left common femoral artery. Weaning seemed to be possible with moderate inotropic agents, but when we lowered CPB flow to $1.5 \mathrm{~L} / \mathrm{min} / \mathrm{m}^{2}$, pulmonary arterial pressure $(78 / 35 \mathrm{mmHg})$ exceeded systemic blood pressure $\left(75 / 40 \mathrm{mmHg}\right.$ ) (PVR; $312 \mathrm{dyne} / \mathrm{sec} / \mathrm{cm}^{5}, \mathrm{Pp} / \mathrm{Ps}$; 1.10 ) and we thus resumed iNO at $10 \mathrm{ppm}$. Weaning from cardiopulmonary bypass then proceeded uneventfully, but pulmonary arterial pressure exceeded systemic blood pressure at the closure of the sternum and during the transfer to Intensive Care Unit. Prostaglandin $\mathrm{E}_{1}(0.02 \gamma)$ was added to lower pulmonary arterial pressure. The postoperative hemodynamics were mostly stable in the intensive care unit. After 12 hours, hemodynamic data remarkably improved (PVR; 167 dyne $\left./ \mathrm{sec} / \mathrm{cm}^{5}, \mathrm{Pp} / \mathrm{Ps} ; 0.75\right)$ and weaning from NO was carried out very gradually on the third postoperative day. The next day, we could discontinue iNO without hemodynamic changes. The patient was extubated on the fourth postoperative day (Fig. 3). After insertion of an inferior venacaval filter, he was discharged from the hospital on the twenty-first postoperative day. The blood gas analysis in normal air at discharge showed pH 7.403, $\mathrm{PaO}_{2} 61.0 \mathrm{mmHg}, \mathrm{PaCO}_{2} 38.0 \mathrm{mmHg}$ and $\mathrm{BE}+0.8$. He takes coumadine and prostacycline 


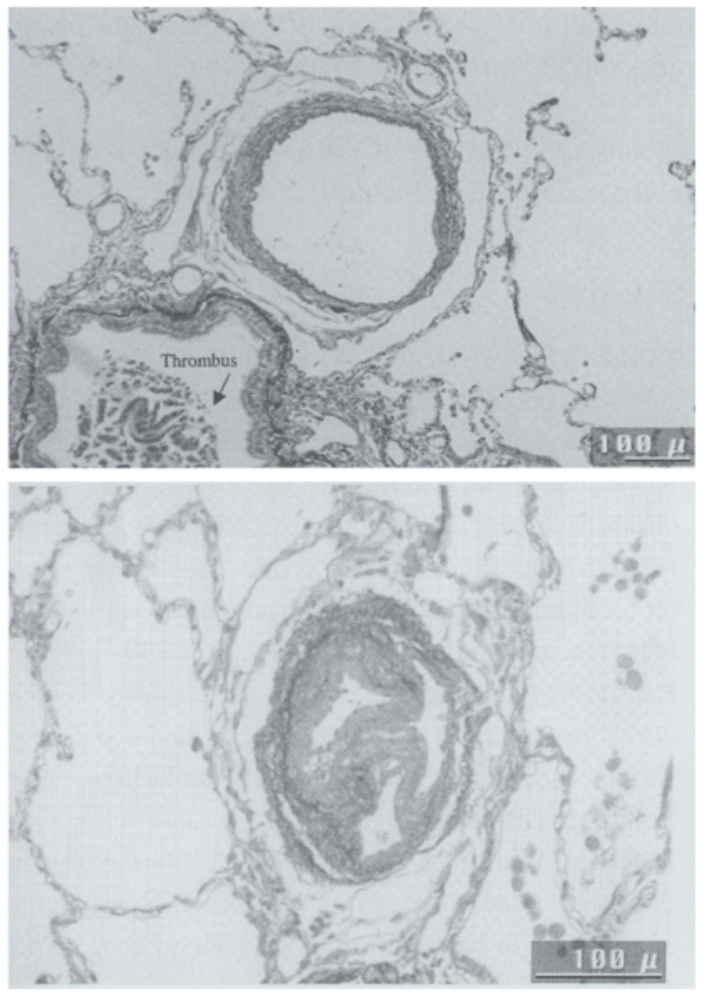

Fig. 2. Histological microphotography showing thrombi almost completely occluding small pulmonary artery and obliterating concentric lamina intimal fibrosis. There were no findings of medial hypertrophy (Elastica-Goldner stained $\times 400$ ).

$(120 \mu \mathrm{g} /$ day) and has remained well with good cardiopulmonary function during six months of follow-up examinations.

\section{Discussion}

In this clinical case presentation, we report the use of inhaled NO as a successful element in the treatment of chronic thromboembolic pulmonary hypertension. Chronic pulmonary hypertension resulting from thrombotic disease carries a poor prognosis, proportional to the degree of pulmonary hypertension. Further pulmonary embolic events were life-threatening to this patient, so we decided to undertake surgery. Although no apparent findings of deep venous thrombosis were found, we decided to insert an inferior venacaval filter after surgery. Most pulmonary emboli arise from the deep veins of the legs. Ultrasonography is positive in 10 to $20 \%$ of all patients without leg symptoms or signs who undergo evaluation and in only $50 \%$ of patients with proven pulmonary embolism. Therefore, the pos-

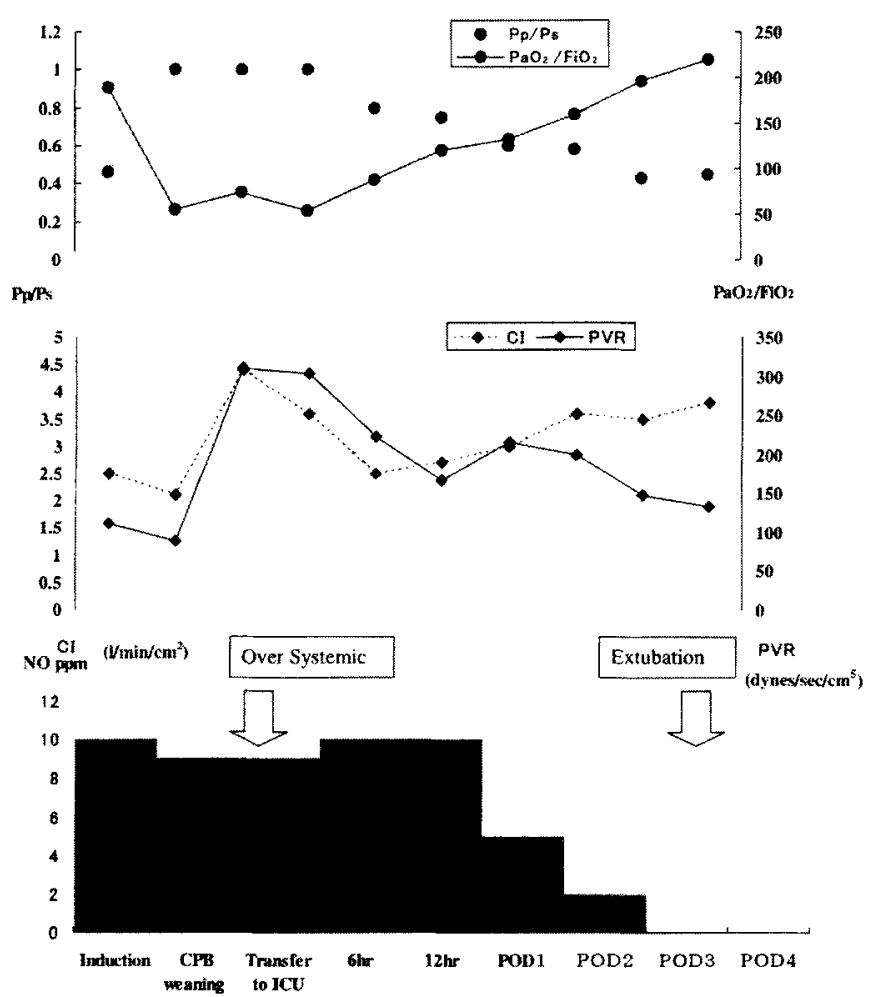

Fig. 3. Clinical course of perioperative period with inhaled NO concentration.

sibility of pulmonary embolism due to deep venous thrombus cannot be ruled out on the basis of negative results on ultrasonography. This is the second reason why we decided on insertion of the inferior venacaval filter after surgery. ${ }^{3}$ About $50 \%$ of the patients with pulmonary embolism who are hemodynamically stable have echocardiographic evidence of right ventricular dysfunction, a finding that is associated with high inhospital mortality. In our case, we can speculate that the cause of the thrombus formation in the right ventricle was blood stagnation due to the acute right ventricular dysfunction at the sudden onset of chest pain. ${ }^{4}$

Hypoxemia and right heart failure due to persistent pulmonary hypertension may be a primary cause of death during perioperative period in such patients. In addition to residual emboli in peripheral pulmonary arteries, vasoconstriction can result from the release of vasoconstrictors by the thrombus or the cardiopulmonary bypass. Hypoxia is one of the most physiologically important pulmonary vasoconstrictors, but the mechanism of hypoxic pulmonary vasoconstriction are unclear. $^{5}$

As already mentioned, inhaled NO causes selective 
pulmonary vasodilatation without decreasing systemic arterial pressure. Intravenous infusion of either prostaglandin $E_{1}$ or nitroglycerin can also reduce pulmonary hypertension but causes marked systemic vasodilatation and can produce hypotension which decreases coronary perfusion of the right ventricle. Recent evidence that the pulmonary vascular endothelium is an important determinant of vascular tone has led to the hypothesis that endothelial injury, secondary to chronic pulmonary hypertension, disrupts these regulatory mechanisms and thereby plays an important role in the development of pulmonary hypertension and its associated increased vascular reactivity. ${ }^{6}$ In consideration of these facts we started admistration of iNO at the induction of anesthesia for prevention of pulmonary hypertension crisis due to stimuli in the intubation period.

Using inhaled NO, a gradual decrease in pulmonary vascular resistance in combination with improved hemodynamics and pulmonary gas exchange was observed in this case. This enabled us to reduce inotropic agents and start the patient weaning from ventilation. The effect of iNO infusion on arterial oxygenation was quantified using the $\mathrm{PaO}_{2} / \mathrm{FiO}_{2}$ ratio. During the clinical course of this patient, the value of $\mathrm{PaO}_{2}$ remained around $70 \mathrm{mmHg}$, but the $\mathrm{PaO}_{2} / \mathrm{FiO}_{2}$ ratio and cardiac index were dramatically improved after CPB weaning. This finding may be explained not only by improvement of hypoxemia but also by improvement in ventilation perfusion matching. Inhaled NO effectively vasodilates well-ventilated regions of the lung. It is suggested that pulmonary vascular endothelial cell dysfunction contributes to pulmonary vasoconstriction after CPB. Inhaled NO can achieve pulmonary vascular smooth muscle relaxation independently of the endothelium. ${ }^{7}$ From this perspective, inhaled NO can be considered to be a useful adjunct to surgical treatment of chronic thromboembolic pulmonary hypertension.

Despite the fact that there are so many reports about clinical use of inhaled $\mathrm{NO}$ as a selective pulmonary vasodilator for pulmonary hypertension, it is accepted minimally use in Japan because of its toxicity. ${ }^{8,9}$ The problem of toxicity is related to NO itself and the formation of nitrate dioxide $\left(\mathrm{NO}_{2}\right)$ by oxidation of NO. In a previous study, very low $\mathrm{NO}_{2}$ room concentrations were recorded during inhaled NO therapy and health care workers were not considered to be at risk. ${ }^{10}$ The second toxicity problem is the production of methemoglobin. Methemoglobin is produced by NO's reaction with oxyhemoglobin. In this patient, concentration of methemoglobin was less than $1.3 \%$ during ICU stay.

In conclusion, low dose inhaled NO is a safe and reasonable alternative to surgical treatment for chronic thromboembolic pulmonary hypertension.

We thank Dr. Shigeo Yamaki for pathological diagnosis and for reading an earlier draft of this paper.

\section{REFERENCES}

1. Palmer RM, Ferrige AG, Moncada SA. Nitric oxide release accounts for the biological activity of endothelium-derived relaxing factor. Nature 1987; 327: 524 6.

2. Girard C, Lehot JJ, Pannetier JC, Filley S, Ffench P, Estanove S. Inhaled nitric oxide after mitral valve replacement in patients with chronic pulmonary artery hypertension. Anesthesiology 1992; 77: 880-3.

3. Kearon C, Ginsberg JS, Hirsh J. The role of venous ultrasonography in the diagnosis of suspected deep venous thrombosis and pulmonary embolism. Ann Intern Med 1998; 129: 1044-9.

4. European Working Group on Echocardiography. The European Cooperative Study on the clinical significance of right heart thrombi. Eur Heart J 1989; 10: 1046-59.

5. Frostell CG, Blomqvist H, Hedenstierna G, Lundberg J, Zapol WM. Inhaled nitric oxide selectively reverses human hypoxic pulmonary vasoconstriction without causing systemic vasodilation. Anesthesidology 1993; 78: 427-35.

6. Steinhorn RH, Fineman JR. The pathophisiology of pulmonary hypertension in congenital heart disease. Artif Organs 1999; 23: 970-5.

7. Wessel DL, Adatia I, Giglia TM, Thompson JE, Kulik TJ. Use of inhaled nitric oxide and acetylcholine in the evaluation of pulmonary hypertension and endothelial function after cardiopulmonary bypass. Circulation 1993; 88: 2128-38.

8. Pinelli G, Mertes PM, Carteaux JP, Hubert T, Dopff $\mathrm{C}$, Burtin $\mathrm{P}$, et al. Inhaled nitric oxide as an adjunct to pulmonary thromboendarterectomy. Ann Thorac Surg 1996; 61: 227-9.

9. Trummer G, Berchtold-Herz M, Martin J, Beyersdorf F. Succesful treatment of pulmonary hypertension with inhaled nitric oxide after pulmonary embolectomy. Ann Thorac Surg 2002; 73: 1299-301.

10. Markhorst DG, Leehoven T, Ulterwijk JW, Meulenbelt J, van Vught AJ. Occupational exposure during nitric oxide inhalational therapy in a pediatric intensive care setting. Intensive Care Med 1996; 22: 954 8. 